\title{
La ilusión de poblar. Territorios, narrativas y mapas del Orinoco y la Guayana en la segunda mitad del siglo XVIII, los umbrales de una geografía humana del porvenir
}

\section{THE ILLUSION OF POPULATING. TERRITORIES, NARRATIVES, AND MAPS OF THE ORINOCO AND GUIANA IN THE SECOND HALF OF THE 18TH CENTURY, THRESHOLDS OF A HUMAN GEOGRAPHY OF THE FUTURE}

\author{
A ILUSÃO DE POVOAR. TERRITÓRIOS, NARRATIVAS E MAPAS DO ORINOCO \\ E DA GUIANA NA SEGUNDA METADE DO SÉCULO XVIII, OS LIMIARES DE \\ UMA GEOGRAFIA HUMANA DO PORVIR
}

Para citar este artículo: Cuevas Quintero, L. M. (2019). La ilusión de poblar. Territorios, narrativas y mapas del Orinoco y la Guayana en la segunda mitad del siglo XVIII, los umbrales de una geografía humana del porvenir.

Perspectiva Geográfica, 24(2), 84-106. doi: 10.19053/01233769.8997

Luis Manuel Cuevas Quintero ${ }^{1}$

\section{Resumen}

Bajo el espacio contemporáneo de las geografías americanas se disponen otros espacios que conforman un gran hipotexto cuya complejidad está integrada por la tradición de los discursos geográficos, a la vez que da cuenta de las formas cambiantes de la percepción territorial. La historia geográfica de estos espacios se organizó e impulsó

1 Doctor en Geografía de la Universidad Nacional Autónoma de México; magister scientiarum en Historia de la Universidad Iberoamericana. Licenciado en Historia con distinción summa cum laude de la Universidad de Los Andes, Venezuela; licenciado en Educación de la Universidad de Los Andes; TSU en Agrotecnia del Instituto Universitario Tecnológico de Ejido. luismanuelvenezuela@hotmail.com https://orcid.org/0000-0003-1468-408X 
por la ilusión de poblar y el discurso del progreso. Este artículo reflexiona sobre este binomio e interpela la historicidad y la geograficidad de los territorios de la cuenca del Orinoco y de la Guayana en el siglo XVIII, de los procesos y fenómenos que los articularon y los hicieron visibles. En tal sentido, narrativas geográficas, mapas y diccionarios constituyeron repertorios que organizaron la imaginación geográfica territorial del Orinoco y la Guayana en el siglo XVIII; su revisión y su crítica sintética es objeto de este trabajo.

Palabras clave: imaginación geográfica, discurso, cartografias, Orinoco, Guayana, historicidad y geograficidad.

\section{Abstract}

Under the contemporary space of the American geographies are other spaces that make up a large hypotext whose complexity is integrated by the tradition of geographical discourses while accounting for the changing forms of territorial perception. The geographical history of these spaces was organized and promoted by the illusion of populate and the discourse of progress. This research work reflects on this binomial and interpellates the historicity and geograficity of the territories of the Orinoco basin and Guiana in the 18th century, of the processes and phenomena that articulated it and made it visible. In this sense, geographical narratives, maps and dictionaries constituted repertoires that organized the territorial geographic imagination of the Orinoco and Guayana in the eighteenth century, its review and its synthetic criticism is the subject of this work.

Keywords: geographic imagination, discourse, cartographies, Orinoco, Guiana, historicity and geograficity.

\section{Resumo}

O espaço contemporâneo das geografias americanas existem outros espaços que compõem um grande hipotexto cuja complexidade é integrada pela tradição dos discursos geográficos, ao mesmo tempo em que explica as formas mutáveis da percepção territorial. A história geográfica desses espaços foi organizada e promovida pela ilusão da povoar e pelo discurso do progresso. Este trabalho de pesquisa reflete sobre esse binômio e interpela a historicidade e geograficidade dos territórios da bacia do Orinoco e da Guiana no século XVIII, dos processos e fenômenos que 
o articularam e tornaram visível. Nesse sentido, narrativas, mapas e dicionários geográficos constituiram repertórios que organizaram a imaginação geográfica territorial do Orinoco e Guayana no século XVIII, sua revisão e sua crítica sintética é o tema deste trabalho.

Palavras chave: imaginação geográfica, povoar, cartografias, Orinoco, Guayana, historicidade e geograficidade.

\section{Introducción: el lugar, los}

\section{lugares, los observadores}

La definición del lugar desde el cual miramos, el locus de enunciación, es importante; sin embargo, este lugar no es uniforme y, en consecuencia, no se limita a una sola perspectiva de observación, sino que contempla diversos puntos de vista de otros observadores que dejan sin efecto la univocidad del discurso sobre el espacio geográfico, sustituyéndolo por una polifonía altergeográfica que está atravesada por una serie de tensiones y de continuidades y discontinuidades que muestran un campo complejo de estudios.

La historicidad (Hartog, 2007) y la geograficidad (Dardel, 1952) del espacio geográfico americano constituyen, en consecuencia, un problema de posicionamiento del observador y de las observaciones que pretenden dar cuenta de un saber espacial cuyas dimensiones espaciotemporales dan cuenta de persistencias y diferencias.

Bajo el espacio contemporáneo de las geografías americanas se disponen otros espacios cuya historicidad y geograficidad puede leerse como un gran hipotexto que da cuenta tanto de las formas cambiantes de la percepción territorial como de las diversas alteraciones de los paisajes geográficos físicos y humanos. Si disponemos estos espacios en una visión de conjunto, de los cuales los más emblemáticos son los de las cuencas del Amazonas, el Mississippi y el Orinoco, podemos reconocer una historia que está organizada e impulsada por la ilusión de poblar y del progreso.

La conexión del pasado geográfico del Orinoco y la Guayana que se estudia en este trabajo no es idéntica con el tiempo actual: si bien se corresponde con un marco geográfico que más o menos sigue siendo el mismo, su visión, en tanto espacio de promisión, expresa diferencias en torno a la consideración de sus recursos, de sus emplazamientos geoestratégicos y de la imaginación geográfica que lo dota de un sentido territorial vinculado a las etnias, a los imperios, las repúblicas y al capitalismo en sus sucesivas fases de articulación.

En este sentido, el problema del territorio y de las territorialidades, concebidos como una forma espacial que teje en sí misma la materialidad y la representación, autoriza nuevos enfoques de la modernidad americana y de sus ilusiones, aciertos y déficits en el campo material de la ocupación efectiva, de la imaginación geográfica y de los documentos cartográficos que dan cuenta de sus dimensiones y de su orientación.

Las perspectivas contemporáneas del pensamiento geográfico y cartográfico redefinen el tratamiento de esa historia y de sus documentos, con nuevos 
giros observables en los corpus de revistas como Terra Brasilis en Brasil o en proyectos como Razón Cartográfica en Colombia, o el llevado a cabo bajo la coordinación de Pedro Cunill Grau en Venezuela, GeoVenezuela, permiten considerar nuevos documentos y nuevos actores en la construcción y la producción de espacios geográficos, es decir, considerar que el espacio ya no solo es soporte de prácticas, sino que es producido en el plano social de la significación y en su materialidad, o construido en el plano del sentido que articula su imaginación geográfica. Es así que los valores de los espacios y su utilidad muestran formas cambiantes de habitar o de expandir la habitabilidad de espacios geográficos otrora considerados marginales. De este modo, materialidad y representación se correlacionan y se determinan mutuamente.

En conjunto, estas premisas inscriben este trabajo en una problemática que considera al espacio en su dinámica, que estudia los diversos registros (cartográficos, narrativos y descriptivos) en los que se plasmó un momento de la experiencia geográfica del río Orinoco y la Guayana.

\section{Umbrales de una geografía humana y geoestratégica en el Orinoco y la Guayana}

De las relaciones humanas con el medio se derivan otros problemas que se agrupan en tres conjuntos: la diversidad de los espacios humanos, el carácter utilitario y la valoración geoestratégica del espacio.

En el siglo XVIII, la construcción de un espacio posible, de una imaginación del paisaje que venía activada por la intervención antrópica y sus nue-

Vol. 24 N. ${ }^{\circ} 2$

julio - diciembre de 2019 pp. $84-106$ vas percepciones en torno a un valor nuevo de los lugares y los recursos contenidos en el medio ambiente, se expresará en los discursos de los religiosos, de los científicos (en especial, de los botáni$\cos )$ y de los ilustrados reformistas.

El principal problema en la óptica compartida de las comunidades de funcionarios españoles y de los misioneros destacados en las zonas especiales lo constituía el poblamiento, factor fundamental en la expansión y estabilización del limes imperial. Es así como la política de fronteras en la Orinoquia se dirigió a la conservación de los núcleos de poblamiento y a la fundación de otros. La Expedición de Límites del Orinoco Marañón (Amazonas) de 1750 a 1760/67 dirigida por José de Iturriaga, Eugenio de Alvarado, Antonio de Urrutia y José Solana y Bote se llevaría a cabo en tal sentido y siguiendo las directrices del Tratado de Madrid de 1750, firmado entre España y Portugal, con varias fundaciones de pueblos y de fortines, sobre todo en el área de confluencia de los ríos Orinoco, río Negro-Guainía y Atabapo ${ }^{2}$ que se sumaban a la preexistente red de asentamientos misioneros que seguían las riberas del Orinoco y de los ríos de los Llanos del Casanare, Meta y Orinoco.

Con anterioridad, y coexistiendo con esta idea, el avance civilizatorio del logos occidental en núcleos de implantación se centrará principalmente en el modelo misional de penetración y ocupación de la tierra ${ }^{3}$, esto al menos hasta la primera mitad

2 Para una información más detallada de esta expedición véase Ramos Pérez (1946), Lucena (1993; 1998) y Lucena y De Pedro (1992).

3 Se estima que los jesuitas poseían al rededor de $222 \mathrm{mi}$ siones solo en Hispanoamérica, ubicadas generalmente en zonas de indios hostiles a la penetración europea (Weber 2007, p. 166; Merino \& Newson, 1995). Los capuchinos, en el bajo Orinoco, fundaron más de 29 pueblos en un lapso de 46 años, entre 1724 y 1770 (Carrocera, 1979). 
del siglo XVIII, y luego bajo un modelo mixto que agregaba fundaciones de presidios, villas y ciudades. La idea de poblar establecía un contraste en la relación espacial de aglomeración y concentración frente a la dispersión: la primera, concebida como un lugar de concentración, era el lugar civilizatorio de ortopedia social; la segunda era el espacio de bárbaros y salvajes, caracterizado por la vida nómada de grupos de cazadores y recolectores en la Orinoquia, y, en menor grado, los de vida mixta.

El modelo occidental no presentaba una unidad de criterio, pues existían opiniones contrarias a las ventajas que las misiones ofrecían. Manuel de Centurión había señalado al ministro de Indias, Juan Arriaga, en 1774, que las misiones dejaban a los indios en una condición tal de inutilidad que en nada se diferenciaba de su anterior vida salvaje (Lucena Giraldo, 1992, p. 71), una percepción compartida por los funcionarios de la administración colonial, quienes pensaban que las misiones solo debían dedicarse a lo espiritual. Sin embargo, pese al episodio de las misiones jesuitas teñido por la sospecha de pretender autonomía territorial, entre otras acusaciones rodeadas de intrigas que ocasionaron su expulsión, el sistema misionero no fue eliminado, sino que fueron debilitadas sus autonomías relativas por las políticas borbónicas de control territorial de las fronteras (Weber, 2007, p. 158).

En el Orinoco y la Guayana, sin embargo, las misiones mantuvieron su fuerza. En los Llanos altos y bajos, y el Caroní y Caura, que forman parte de la cuenca del Orinoco, las misiones de franciscanos, agustinos y capuchinos catalanes experimentaron una expansión eficaz y sostenida sobre los restos de las misiones jesuitas (Donís, 1997; Carrocera, 1979; Weber, 2007). También, junto a este proceso de poblamiento, los viajes de exploración misionera continuaron abriendo conexiones entre espacios ${ }^{4}$. Un ejemplo de ello lo constituyen las exploraciones de los capuchinos en el bajo Orinoco, el Cuyuní, el Barima y el Parime.

La expansión al sur del río Caroní, el gran afluente del Orinoco medio, condujo a la fundación de la Barceloneta (La Paragua) en 1770 por Fray Benito de La Garriga. Los capuchinos también emprendieron expediciones hacia el Parime remontando el Paragua con el mismo La Garriga y Tomás de Mataró. En una segunda expedición con Félix Vic lograron vencer los obstáculos naturales de gran parte de la zona. No obstante, ambas exploraciones de reconocimiento se detuvieron en las barreras montañosas de la sierra de Pacaraima.

De ese proceso, varios trabajos cartográficos como el mapa anónimo de misiones capuchinas de 1735 , los de Fray Carlos de Barcelona: "Misiones capuchinas catalanas y Anathomía Geográphica" de 1771 y el mapa de 1779 (Figura 1) y, finalmente, otro mapa anónimo de "Misiones Capuchinas de la Guyana de 1789", se constituyen en un factor importante por cuanto muestran las proyecciones hacia el sur y hacia el sureste de la cuenca del Orinoco y revelan además que parte de los movimientos hacia la terra incognita y de sus registros visuales primarios iban de la mano de los religiosos ${ }^{5}$. Estos

4 Los capuchinos catalanes Ilevarían con fray Antonio La Garriga una política exploratoria en el Alto Caroní, El Paragua, Barima y Cuyuní, una labor que coincidía con el despliegue misionero en otras partes de América como Nuevo México, río Bermejo, los Andes orientales en su conexión fluvial con el Amazonas y la ruta Chiloé-Patagonia entre 1776 y 1792 (Weber, 2007, p. 203). En este sentido de la movilidad misionera, los mismos jesuitas se fueron convirtiendo en prácticos de las zonas geográficas olvidadas u omitidas y fueron conocidos por ser los hombres de los ríos (Del Rey, 2011).

5 Parte de estos mapas se pueden consultar en la web David Rumsey Historical Map Collection https://www.davidrumsey.com/ 
mapas, además, se suman a los elaborados por la orden jesuita (Del Rey, 1975; Cuevas, 2012) y eran un indicador de las coberturas espaciales, así como una ventana a las posibilidades socioeconómicas de esos territorios de los márgenes y de los progresos del modelo de poblamiento.

En medio de este proceso poblador, la lógica imperial de jerarquización del espacio subalternizaba las etnias bajo categorías de salvajes y bárbaras. Pese al registro etnográfico de la geografía humana del Orinoco, hecho por religiosos, no se lograba reconocer en la mentalidad reformista lo que durante miles de años las etnias que habitaban el Orinoco habían diseñado, es decir, sus propias respuestas al medio que les permitían explotar, como hemos visto en el caso del delta, las posibilidades que este ofrecía. Eugenio de Alvarado, uno de los miembros de la Expedición de Límites al Marañón/Orinoco (1750-1760), destacado para hacer observaciones en el bajo Orinoco, el Cuyuní y el Esequibo, diría del Delta:

Todo un país anegadizo, aunque frondoso, de corpulentos árboles y palmas, en especial de mayo a diciembre, que es la estación de las crecientes, pero no por eso dejan de habitarle en todos los tiempos más o menos número de indios infieles pacíficos de la nación Guaraúna, que gustan de parecer en estas selvas anfibios racionales (1999, pp. 153-154).

Jean Nicolás Bellin, cartógrafo e hidrógrafo del Ministère de la Marine de Francia, quien elaboró mapas sobre el curso del Orinoco y las Guayanas apoyándose en la historia de Gumilla y en datos suministrados por los marinos exploradores de las costas guayanesas desde la Cayyenne al Esequivo y la Guayana española, revelaba al mundo académico de París en 1763 la fertilidad de la región. Para ello, la asociaba con la existencia de una red compleja de ríos que la irrigaban, favoreciendo el paisaje de "bosques" (selvas) que ofrecían condiciones para el aprovechamiento de recursos, la producción y el sustento, de modo que las selvas del Delta:

Comunican al terreno una humedad que vuelve los valles por donde pasan de una fertilidad sorprendente; las llanuras están cubiertas de una cantidad prodigiosa de árboles de toda especie, que forman el más bello paisaje que pueda verse y que son de un grosor desconcertante (Bellin, 1986, p. 45).

Se explica entonces que una de las grandes preocupaciones será dar cuenta de las bondades de la geografía física que trocaba la antigua percepción de la humedad como ambiente degenerativo a la humedad como ambiente de fertilidad. Sin embargo, frente a estos pueblos "anfibios" de grado civilizatorio bajo, se oponía la agricultura como indicador occidental de cultura, una cuestión presente en las observaciones de jesuitas como Rivero, Gumilla y Gilij, quienes también habían elaborado sus respectivos discursos de la abundancia y de los niveles de desarrollo civilizatorio según apareciese o no el trabajo de la tierra (Cuevas, 2012; 2017b).

En muchos casos, esa vida de las etnias orinoquenses que los occidentales percibían como nómada no era sino una consecuencia de modos de vida adaptados a las condiciones ecológicas diversas y a los periodos de lluvia y de sequía. Estos grupos eran nómadas estacionales o en algunos casos mi- 
grantes en función de los ciclos de disponibilidad de los recursos ${ }^{6}$. En consecuencia, y dada la movilidad territorial y ecológica, la labor de aglutinar a estas poblaciones se hacía más difícil. Por algunos momentos, el espacio de la misión se diluía en fugas, rebeliones, epidemias y hambrunas que atentaban contra su estabilidad, por ello la solución para los religiosos fue crear unidades de producción ganadera que abastecieran a los asentamientos misioneros, asegurando así la sostenibilidad alimentaria. Bajo su impulso se logró al menos estabilizar algunos núcleos de poblamiento. Frente al espacio imaginado de la barbarie, los ilustrados llevaban la voz "universal" del progreso y la felicidad a los pueblos. El carácter utilitario del nuevo espacio en términos de hábitats nuevos para el poblamiento europeo suponía su acondicionamiento en modelos de ciudades, villas y presidios, a los que se sumaban las políticas de infraestructura portuaria para el caso de asentamientos situados en las riberas de los ríos, y en la construcción de reductos defensivos.

En el fondo, las misiones también tenían este carácter de ocupación y acondicionamiento de los lugares. Los misioneros jesuitas y los capuchinos ocupaban enclaves estratégicos en el curso del río Orinoco y en sus principales afluentes, como, por ejemplo, los emplazamientos de Atures y Maipures, el de Cabruta, el de Moitaco y el del Caroní-Paragua de los capuchinos ${ }^{7}$.

6 Para el caso de la pampa argentina, Mandrini (1992) ha mostrado estos errores de percepción sobre un nomadismo ficticio; otro tanto podemos observar con la relación de los ciclos de recolección, caza y pesca que forzaban a dirigirse en grupos a determinados nichos ecológicos. Denevan (1992) también ha rebatido la idea de una naturaleza prístina asociada a la idea del "salvaje natural" y, por el contrario, ha mostrado que muchas selvas poseían un origen antrópico.

7 Para una explicación de la función defensiva y militar contenida en la denominación de reducción adaptada para
A la par de esta preocupación por la localización de los emplazamientos se elaboraban discursos en los que se trataban de hacer atractivos estos espacios para la inmigración europea, una labor que tenía su antecedente inmediato en Rivero, pero sobre todo en Gumilla (1983), quien a mediados del siglo XVIII en 1741 y 1745 había mostrado extensos inventarios de recursos en su obra, cuyo largo título muestra, de por sí, las dificultades de un ensamblaje en el que se mezclan ciencia y fe: El Orinoco ilustrado y defendido, historia natural, civil y geográfica de este gran río y de sus caudalosas vertientes. Gobierno, usos y costumbres de los Indios sus habitadores, con nuevas y útiles noticias de animales, árboles, frutos, aceytes, resinas, yervas, y raices medicinales; y sobre todo se hallarán conversiones muy singulares a nuestra Santa Fé... (sic).

Junto al trabajo constante de registros que dan cuenta de una percepción de la abundancia y la exuberancia de paisajes pródigos, expresada en los inventarios naturales que contienen los textos de los religiosos, la mirada ilustrada en la Orinoquia tenía en el propio carácter de la Expedición de Límites de 1750/54-1760: el objetivo de observar la naturaleza de un modo más sistemático y acorde con las enseñanzas de Linneo, una labor encomendada a Per Löefling, quien trabajó en el área del bajo Orinoco y el Caroní.

De este modo, la existencia de recursos (sobre todo de suelos fértiles y productos botánicos que podían alimentar a las economías locales y metropolitanas) fue señalada como recursos potenciales $y$, en el caso del legado de Löefling, estos fueron clasificados de acuerdo con el método moderno de taxo-

las misiones en el contexto violento de las fronteras, véase Cuevas (2006) y Lacotoure (1993). 


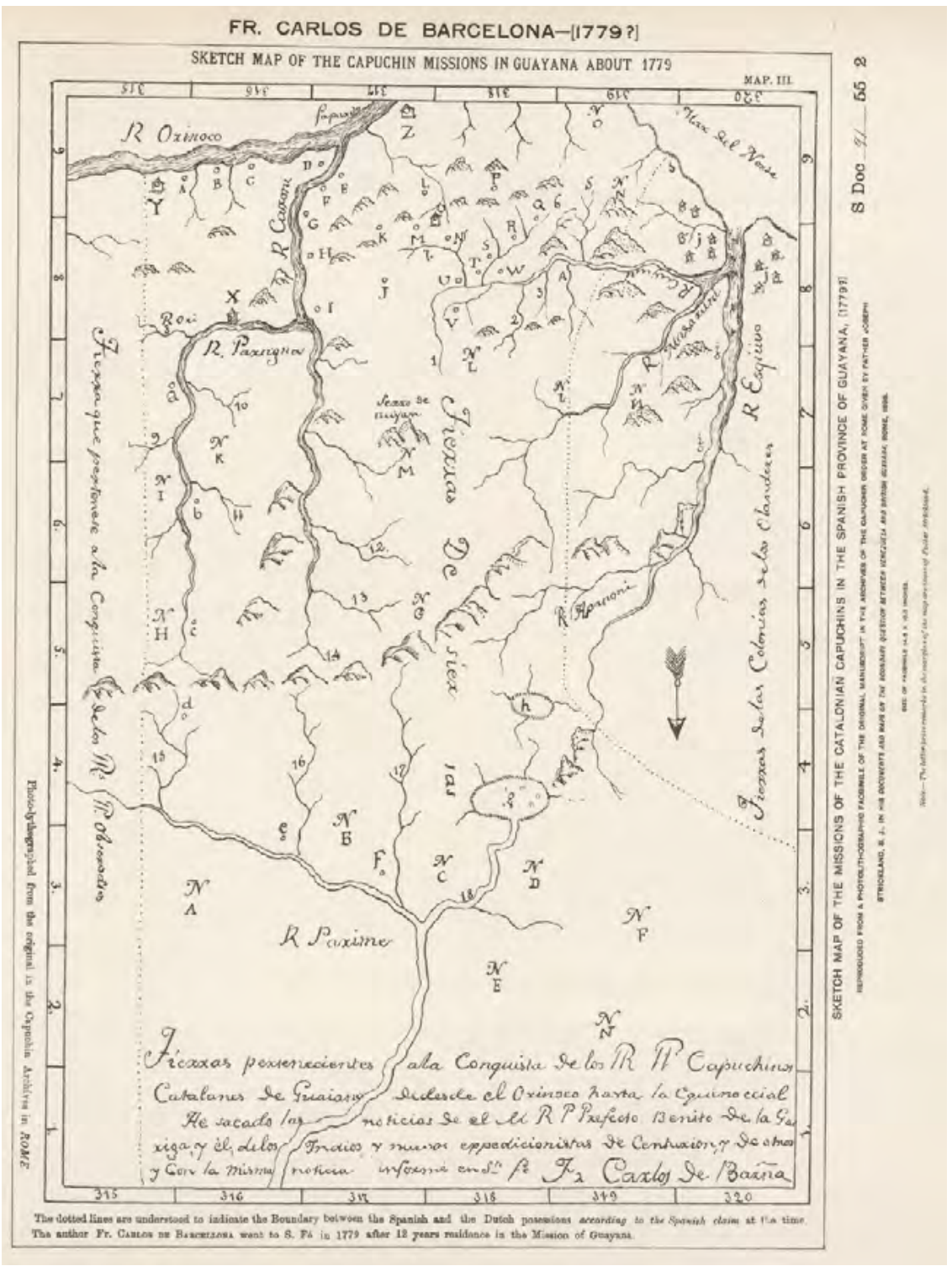

FIGURA 1.

Sketch Map of the Missions of the Catalonia Capuchins in the Spanish Province of Guayana de Fray Carlos de Barcelona (1779),

Fuente: David Rumsey Historical Map Collection. nomías linneanas, mostrando así un nuevo espacio para el coleccionismo botánico de la ciencia en la Orinoquia. Con él y con otros discípulos de Linneo se iniciaba una gran red de globalización botánica que tendría sus centros de recepción y cálculo en los jardines botánicos europeos y en los gabinetes $\mathrm{y}$ museos de ciencias naturales, que formaban parte de prácticas localizadas y conectadas (Amodio, 1998; Lucena, 1998; Ryden, 1957; Livingstone,
2002; 2004). No obstante, la muerte temprana del botánico sueco en las riberas del Orinoco arrojaría por la borda el proyecto de sistematización.

La mirada sobre los recursos también estaba gobernada por el carácter utilitario y geoestratégico. Parte de las exploraciones en el alto Orinoco se habían hecho también con el ánimo de reconocer la existencia de cacahuales (cacao silvestre), el "oro vegetal" de la política borbónica del siglo XVIII. 
Hacia el último tercio de este siglo, las exploraciones se habían concentrado en una política de contención del avance holandés en la zona del Cuyuní y de fortalecimiento del bajo Orinoco. José Inciarte (1968), quien había hecho viajes hacia el suroriente de la Guyana y el sur del Delta entre 1779 y 1783, hablaba de planes para desarrollar el bajo Orinoco y la costa atlántica del Esequibo con la finalidad de estabilizar una zona que lucía desguarnecida, una labor que debía acompañarse con planes de activación económica ganadera y forestal que asegurasen la estabilidad de los nuevos poblamientos proyectados.

De esta manera, las exploraciones para determinar emplazamientos adecuados para el poblamiento al sur de la Boca Grande en el Delta del Orinoco, y en la zona del Barima, frenarían el avance holandés desde Berbice y Demerara, garantizando el dominio imperial.

Este proyecto seguía la dirección que el intendente José de Ábalos y la administración férrea de José de Gálvez, ministro de Indias, les había impuesto. Se trataba de revitalizar el bajo Orinoco mediante la ganadería y para ello era necesario encontrar llanuras aptas para tal propósito, cuestión que no sucedió porque la exploración no logró hallar zona de sabanas aptas para tal fin (Lucena Giraldo \& De Pedro, 1992). Muchas de las zonas del bajo Orinoco eran inundables y pantanosas, y en consecuencia el proyecto ganadero no pasó de ser más que una ilusión enfrentada con las condiciones ecológicas y climáticas del medio tropical lluvioso.

Todo este conjunto de intereses y de problemas espaciales a resolver se explican también en el giro de la geografía política del dieciocho. Entre 1731 y 1751, en un lapso de 20 años, los principales obstáculos podían identificarse del modo siguiente: el área de Guayana se convertiría en un espacio caótico caracterizado por el retroceso de las misiones jesuíticas (Del Rey, 1998); las tensiones territoriales entre órdenes religiosas por áreas de acción ocasionaban frecuentes disputas; los asaltos y razzias de Caribes y holandeses, asociados para el suministro de esclavos al Caribe y a las plantaciones guayanesas, y el avance portugués por río Negro eran factores importantes. A ello se sumaba el conocimiento parcial y fragmentado del espacio geográfico que impedía formarse una idea totalizante de lo que significaba el territorio articulado por la cuenca del Orinoco. Esto suponía una constante presión por elaborar mapas y planos confiables que mostrasen una dimensión concreta de la soberanía imperial en la Guayana.

El Orinoco se convertía en un espacio perturbador para la administración hispánica metropolitana y para las administraciones locales asentadas en las ciudades región vinculadas al Nuevo Reino de Granada y las gobernaciones de Cumaná, Guayana, y luego de las políticas reformistas, a la Capitanía General de Venezuela y la Real Intendencia de Caracas.

Entre 1755 y 1760 , una serie de exploraciones de la Expedición de Límites reconocerán distintos espacios:

a) El bajo Orinoco, el Esequibo, Barima y Caroní, con Eugenio de Alvarado en 1755, con la finalidad de evaluar las misiones capuchinas y las rutas de holandeses y Caribes.

b) Ruta hacia el alto Orinoco atravesando los raudales de Atures y Maipures, con Solano y Bote.

c) Ruta del Apure-Sarare con V. Doz y Guerrero en busca del piedemonte andino de la Provin- 
cia de Mérida en 1757-58 para abrir el derrotero de los llanos bajos del Apure y Barinas.

d) Ruta del Guaviare hacia San Fe de Bogotá con Alvarado en 1760.

e) En 1759-1760, yendo hacia el sur por río Negro, entrarán en contacto en Mariuá (Barcelos) con la avanzada portuguesa Francisco Fernández de Bobadilla y Simón Santos, lo cual reforzó el descubrimiento de la conexión entre cuencas (Orinoco-Amazonas) que con anterioridad había hecho el misionero jesuita Manuel Román y que La Condamine había mostrado en Francia.

f) Finalmente, las exploraciones de Díez de la Fuente y Fernández de Bobadilla en el alto Orinoco entre 1759 y 1760 tuvieron como consecuencia un giro definitivo de orientación geográfica del río Orinoco y sus fuentes hacia el sistema de sierras de la Guayana profunda; con ello se desplazó la idea y la percepción anterior, sostenida por jesuitas como Gumilla (1983), de la existencia de un Orinoco andino.

En la visión borbónica del territorio, se trataba de ejercer un dominio espacial que articulase una red entre la metrópoli peninsular, las ciudades región americanas y los espacios interiores. Esta situación obligaría al imperio a redefinir su presencia (Brendeke, 2016; Weber, 2007; Lynch, 1987) bajo un proceso de transición de concepciones económicas ahora ligadas al proyectismo borbónico (Ludlow \& Martínez, 2007).

En este contexto, el impulso de expediciones hacia fronteras mal definidas como la de la Orinoquia, con el objeto de fijar el limes imperial, se hizo dentro de criterios más científicos, objetivos y realistas, de utilidad para la "ciencia de gobierno".
Estrategias de modernización reformistas vinculadas a una práctica que operativamente articulaba saber y poder, ciencia y Estado, se reflejarían en el propio tratado de Madrid en 1750, que impulsó la Expedición de Límites del Marañón/Orinoco. Esta expedición había llegado a Cumaná y de allí había partido hacia la Guayana en dos frentes: uno por la ruta terrestre siguiendo la vía ganadera y de misiones, y otro bordeando la costa con dirección al Delta y a la Boca Grande para acceder al río Orinoco (Cuevas, 2017b). La expedición, si bien no logró el objetivo de demarcar el territorio al sur del río Negro, produjo una documentación que atrajo el interés de la Corona española hacia la Orinoquia, que fue asociada a las políticas reformistas traducidas en planes de fortificación y en el diseño de una red espacial de pueblos que aseguraban la presencia hispánica en el curso de la importante cuenca del norte de la América del Sur. Esta acción se complementaba con el esfuerzo que las órdenes venían haciendo para atraer los ojos imperiales al Orinoco en un momento de redescubrimiento de esa geografía fluvial.

Entre 1776 y 1790 se estudiaron en el bajo Orinoco las posibilidades madereras del área, que incluyeron a los prestigiosos ingenieros navales Diego Seaman y Charles Smith. Entre 1789 y 1791 se estudiaron Upata, Chirica, Santa Ana de Puga y Angostura (Lucena, 1999, p. 35). Estas áreas, hoy día, están vinculadas a provincias geológicas de alto valor en recursos minerales como hierro y oro.

En 1788 el ingeniero militar y gobernador de la Guayana, Miguel Marmión, organizó la exploración de los ríos Curumo y Cuyuní al mando de Antonio López de la Puente (Donís, 1997; Perera, 2006). En ese mismo año, este funcionario ilustrado elaboró una Descripción corográfica-mixta de la provincia de Guayana en que se da razón de 
los ríos que la bañan y facilitan sus comunicaciones: de su población, tierras de labor útiles, de sus frondosos montes, frutos y comercio, y se proponen algunos medios los más asequibles y conducentes a su verificación y aumento (Marmión, 1999). Un texto paratáctico que contenía toda una proyección de las condiciones de poblamiento.

Este documento (así lo muestra el propio enunciado de la obra) puede ser considerado como una síntesis de los problemas centrales para el último tercio del siglo XVIII referidos a planes de defensa, poblamiento, aprovechamiento de recursos, prospecciones interiores $\mathrm{y}$, sobre todo, el reconocimiento del Orinoco como una llave geográfica clave del eje comunicacional del norte de la América del Sur y del espacio interior que bifurca la comunicación con los Andes y con la zona norte del Amazonas. En 1792 se colocaría en la cuenca del Cuyuní una posta para frenar las incursiones holandesas y proteger los pujantes poblamientos ligados a las misiones capuchinas y sus hatos ganaderos. El mismo Marmión, en 1794, dibujaría un "Plano del río Orinoco desde sus bocas hasta la capital de Guayana" que ofrecía detalles que el mapa de Centurión, "Plano general de la provincia de Guaiana, que, con la exactitud posible y respecto de su dilatada circunferencia e incógnito centro, ha formado con las noticias adquiridas hasta oy $31 \mathrm{de}$ diziembre de 1770 el comandante general de ella", no lograba visualizar. El ciclo costero de exploraciones y dibujo de proyectos en la Guayana atlántica (y con ello el del Delta y el bajo Orinoco), que era una respuesta a la presión de potencias rivales de España en el contexto de una geografía política en disputa por el control de las vías de acceso fluviales, finaliza, si seguimos a Lucena (1999, p. 36), con las noticias de José Luis Basanta en 1788 y Rafael Mas en 1802, que se recogerían en la Memoria de posiciones hidrográficas de Felipe Bauzá, impresa en Londres en 1827, y luego en su "Mapa de Colombia" de 1841 en el que, además, reconocía en una nota los aportes de Inciarte, Solano y Bote y otros ingenieros militares sobre los derroteros del Orinoco.

El conjunto de estas preocupaciones en una visión de campo y perspectiva se lee mejor en un contexto de inserción de los lugares y de los fenómenos geográficos dentro de la lógica de la geografía política imperial.

En este sentido, la nueva situación espacial que reflejan los documentos trataba no de una mera suma de partes, sino de un complejo tejido de observaciones e imágenes de una geografía física y humana diferente, un producto del movimiento dialéctico de la experiencia de la biblioteca en la que habían sido formados los funcionarios y los misioneros confrontada con la experiencia del recorrido geográfico y el flujo de esas observaciones en los centros de recepción o cálculo.

\section{El giro discursivo: la cartografía de Cruz Cano y Olmedilla y el diccionario de geografía de América de Antonio Alcedo}

Los problemas geográficos que afrontaron los observadores religiosos e ilustrados del Orinoco muestran la formación de una conciencia geográfica que responde a un contexto de transición en el campo del saber y de las relaciones prácticas con el espacio. Los textos del siglo XVIII comparten un carácter fundacional para el imaginario geográfico 
y para la propia práctica científica abierta a mostrar lo no conocido ${ }^{8}$.

No solo los obstáculos geográficos naturales, sino la existencia de un mosaico de pueblos de escasos hábitos sedentarios y de un contexto demográfico disperso y de baja densidad en función al espacio de ocupación estimado para el momento, se convirtieron en factores que detenían el proceso de implantación colonial fundado en el binomio del saber-poder sobre el espacio. A esto se sumaba una percepción de riesgo en tierras inhóspitas y el escaso valor que tenían sus productos, al menos para el sistema de gustos y demandas del momento, cuestión que comenzaría a revertirse en el siglo XVIII al calor de un nuevo discurso del progreso.

Esta situación explica el giro discursivo hacia un espacio gobernado por la imagen geográfica de la abundancia. La diferencia con el régimen discursivo anterior, que se movía entre una percepción de los espacios periféricos y de frontera ligada a la imagen del espacio sagrado y de la barrera selvática y desértica (Cuevas, 2017a), radica en que ahora el nuevo discurso se vincula a dos funciones: por un lado, la defensa de un espacio, y por otro, la apertura a opciones de aprovechamiento, como sucedía en las áreas estabilizadas ${ }^{9}$. El es-

8 Una situación análoga sucedía con respecto al desconocimiento de gran parte de Europa con respecto a la propia península ibérica, y puede leerse en el contexto que produjeron los nuevos diccionarios geográficos de España. En 1795. Peré y Casado y Antonio Vegas, en las adiciones y correcciones que hicieron en reediciones del diccionario de Juan de la Serna de 1750 que, a su vez, había sido realizado a partir de los prestigiosos diccionarios geográficos de Echard, The Classical Geographical Dictionary de 1715, y de Ladvocat, Dictionnaire géographique portatif de 1743, expresaban la necesidad de hacer frente a la ignorancia que los extranjeros de otras naciones europeas tenían acerca de la geografía española (Capel, 2003, p. 113)

9 Las áreas americanas de mayor estabilidad y con mayor densidad demográfica eran las mineras, así como las zonas costeras y algunos espacios agrícolas y ganaderos que la pacio paratáctico se superpone sobre el liminar y gobierna las visiones geográficas, sus “diseños y sus designios" en proyección hacia las periferias (Bueno, 2011).

La estabilización relativa de las misiones en la Guayana hacia 1730 inicia un nuevo derrotero para la configuración de una imagen más atractiva de esos espacios, motor de los intereses de reordenamiento imperial bajo el que se construyeron dos de los sentidos de relación sensorial y material referida al discurso de la abundancia: una de carácter religioso, vinculada a un código bíblico (Cuevas, 2012; 2017a), y otra contenida en un código imperial utilitario de la ciencia ilustrada. Sobre el discurso impregnado de teología natural en transición se fundará dialécticamente el discurso utilitario del reformismo y el proyectismo contenido en la documentación de los funcionarios del siglo XVIII, situación que afectará el tratamiento de los problemas geográficos dentro de un diseño de cálculo cuya realización concreta, la más de las veces, fue precaria, a pesar de que algunos núcleos de poblamiento se mantuvieron en el tiempo al sobrevivir a los factores de reacción violenta de los grupo étnicos en resistencia, entre ellos los Caribes, y de que la red de defensa integrada por los fortines San Francisco de Asís, San Gabriel y San Diego de Alcalá, en el bajo Orinoco, ofrecían cierta seguridad en el acceso hacia el interior. Juan Antonio Perelló haría un importante trabajo de levantamientos topográficos y de planos de los fortines y de la Provincia de Guayana entre 1760 y 1780 (Capel et al., 1983, p. 370).

propia implantación había transformado en función de las demandas de mercado. Por ejemplo, las zonas de ingenios de caña de azúcar, las plantaciones de cacao y los fundos o estancias ganaderas para el caso de la Tierra Firme. 
En el contexto general, se observa un cambio, pero también una coexistencia (al menos en el discurso geográfico religioso) en la percepción de los espacios físicos y humanos de visiones providencialistas del espacio con otras ligadas a un proyecto de saber-poder fundamentado en la razón. Las prácticas de observación de los lugares transitan tenuemente hasta marcar diferencias entre la concepción cristiana que proyectaba en esos espacios el carácter sagrado de la creación divina dispuesta al hombre (Cuevas, 2006; 2012) y la que resignifica el espacio racionalmente bajo el reino de la ciencia nueva, con largos inventarios de especies y sus incipientes referencias a las zonas y al medio en el que podían encontrarse, lo no conocido de una geografía difícil en función de los accidentes geográficos y la delimitación, y la barbarie a modelar según criterios de racionalidad.

Sobre esta tríada de las ideas ilustradas se autorizará la acción occidentalizante, transición que construirá una nueva territorialización de un campo para el saber y de áreas que ocupar y "redimir" mediante la imaginación geográfica y la implantación de una nueva relación entre el hombre y el medio, cuya representación, además, se apoyaba en discursos y cartografías de naturaleza múltiple que siguen mostrando horizontes para la expansión. Los centros de cálculo organizaban la información en mapas sintéticos y diccionarios geográficos. Como corolario de este proceso de globalización de los lugares, conviene detenernos en dos ejemplos referentes a estas formas de expresión geográfica del conocimiento, de su apropiación y de sus escalas.

La cartografía de Juan de la Cruz Cano y Olmedilla debe entenderse en el contexto de las disputas por los dominios americanos. Sus mapas ${ }^{10}$ enla-

10 Su ficha biográfica puede consultarse en web de la Bi- zan de una forma clara la relación del Orinoco y la Guayana dentro del esquema imperial de representación, en un contexto que resume la política territorial reformista que dominará el discurso geográfico de la segunda mitad del siglo XVIII. Este geógrafo se había formado junto a Tomás López en la escuela cartográfica francesa de Jean Baptiste Bourguignon d'Anville, geógrafo real, quien había mapeado los cuatro hemisferios y formaba parte de los emergentes círculos de la ciencia ilustrada. Bajo su influencia, Cano y Olmedilla elaboró una serie de mapas, dentro de los cuales se destaca el Mapa geográfico de América Meridional que luego sería ampliado en los llamados mapas murales, integrados por ocho grabados ${ }^{11}$. El mapa mural encargado por la Secretaría de Estado en 1764 se terminó de confeccionar luego de diez años de trabajo, en 1775, bajo el mismo título. Había sido hecho con un cuidado meticuloso en las fuentes y solo indicaba las informaciones exactas, entre ellas las suministradas por la Secretaría de Indias y las contenidas en el atlas de Bellin, con los ajustes de la actualización cartográfica de los depósitos oficiales. De esos grabados o planchas nos interesan los números 1 y 2 .

La primera plancha o grabado muestra el área correspondiente a las divisiones político-administrativas de la parte centro y norte de la América del Sur y destaca el Nuevo Reino de Granada y la Provincia de Nueva Andalucía, así como un recuadro referido al puerto del Callao en el reino del Perú (Figura 2). En sus detalles geográficos, el mapa refleja el cambio de orientación del Orinoco, cuyo nacimiento ahora aparece vinculado hacia el orien-

blioteca Histórica de la Universidad Complutense: http:// biblioteca.ucm.es/foa/55961.php.

11 Este mapa mural compuesto de ocho grabados puede consultarse en la Biblioteca Digital Hispánica: http://bdh.bne. es/bnesearch/detalle/bdh0000051497. 
te, hacia el lago Parima, y establece de forma clara la estratégica comunicación entre los ríos Negro y Orinoco a través del Casiquiare con una etiqueta explícita.

No obstante, Cruz Cano y Olmedilla, a pesar de aspirar a ofrecer informaciones exactas, señala en este grabado la existencia del lago Parima y su vinculación como fuente del río. Este grabado es además interesante porque agrega otras toponimias al mismo lugar, como Paranapitinga y mar Blanco, y sobre el emplazamiento del mítico lago se superpone una pequeña etiqueta de "caribes antropófhagos", lo cual muestra la persistencia de una división de la geografía humana entre civilizados y bárbaros, usualmente relegados a los espacios ignotos como los del lago Parima, hacia donde se ubicaban las cabeceras del río Orinoco.

El segundo grabado de ese mapa mural (Figura 3) refiere a la Guayana y contiene un recuadro en la margen derecha, en la que se ensambla un plano detallado de Angostura, titulado:

Plano del sitio de la Angostura donde se ha establecido el Quartel Gral. De R. Orinoco, la población de la Nueva Guayana y Fortaleza de S. Gabriel, construida de orden de S.M. y dedicada al S. Sr. D. Gabriel Antonio Nro. Infante, por disposición de D. Juan Moreno de Mendoza, Governador de dichos establecimientos.

El plano, elaborado en la administración guayanesa de Joaquín Moreno de Mendoza entre 1764 y 1766, fue ensamblado por Cruz Cano y contiene una serie de informaciones muy importantes con referencia a los lugares habitados, iglesias, emplazamientos, hatos ganaderos y fortalezas. Entre los datos que ofrece, llama la atención el referente al comportamiento del río Orinoco con respecto a los usos de los puertos fluviales, donde distingue los puertos de verano, de diciembre a marzo, y de "Ybierno" (invierno), de junio a septiembre; los otros meses quedaban indeterminados, pues configuraban los intervalos de transición en el lento crecer y decrecer de ríos de tal envergadura, un régimen que obviamente no se podía precisar de forma exacta y que afectaba los intercambios comerciales a lo largo del río y en conexión con el mundo atlántico.

Este mapa, sin embargo, muestra un conocimiento claro de la hidrografía, de los ritmos de las crecientes y decrecientes del Orinoco ligadas a los regímenes estacionales de lluvia y sequía. El documento cartográfico traducía una parte de los problemas geográficos inscribiendo al río en una perspectiva de mayor amplitud, que marca un pasaje de la geografía del Orinoco a un nuevo régimen de la imaginación geográfica y los flujos de productos coloniales. El mapa, a la vez que sintetiza una gran parte del conocimiento geográfico del Orinoco, seguía mostrando el espacio del deseo que movilizaría en los siglos venideros el valor del descubrimiento de los lugares como tema de un régimen de la historia de la geografía.

La observación y la valoración de lugares expandieron los horizontes del conocimiento geográfico en función de una cobertura física e imaginaria, que habilitaba una red más densa de informes y de descripciones. La activación del proceso dialéctico construyó una relación uno/partes de una imagen geográfica regional que se articulaba con el espacio imperial y su geografía diversa. Surgía un Orinoco no limitado, sino abierto al problema del conocimiento universal, multiescalar, $\mathrm{y}$, sin embargo, la inmensidad del espacio seguía retando a los observadores. 
Esto se explica mejor en el contexto general de América. El optimismo del saber del continente por las noticias, dentro del binomio de un territorio, una monarquía, puede observarse en el Diccionario geográfico-histórico de las Indias Occidentales o América: es a saber: de los Reynos del Perú, Nueva España, Tierra Firme, Chile y Nuevo Reyno de Granada de Antonio de Alcedo. Este geógrafo, nacido en la Audiencia de Quito, era hijo de Dionisio de Alcedo, que había otorgado una de las licencias de publicación del Orinoco ilustrado $y$ defendido de J. Gumilla, reconociendo así su valor geográfico e histórico. Para Antonio de Alcedo, la intensa movilidad del conocimiento geográfico en función de recabar noticias de los espacios interiores era el principal indicador de incompletud de toda síntesis y promesa de futuro para la expansión del saber dentro de un cuerpo que se abría para una segunda fase de expansión.

En su Diccionario geográfico-histórico, compuesto por más de mil entradas de toda América, se recogen importantes informaciones del Orinoco (1788, vol. III, pp. 389-391), de sus características fisiográficas y humanas, su dirección y sus accidentes, de las facilidades y dificultades para su navegación motivada por los periodos de lluvias o verano y la presencia de los raudales como un obstáculo para el acceso al alto Orinoco y, con ello, hacia la parte norte del Amazonas-Río Negro. En su exposición, el espacio toma un valor geográfico dominado por la extensa red hidrográfica de sus afluentes, que favorecen las comunicaciones internas y con el Caribe y el Atlántico.

En otras entradas describe sintéticamente los ríos que forman la hoya hidrográfica y da cuenta de sus paisajes de deleite, como, por ejemplo, el río Caroní, uno de los principales afluentes guayane- ses del bajo Orinoco, o del horror, como sucede al describir al caudaloso Caura y su geografía, asediada por las razzias de Caribes y holandeses, o de la desolación producto de la miseria, como sucede con la languidez del paisaje de las decadentes Ciudad Real, Real de Corona y el pueblo de San Fernando de Maipures, todas fundadas en las orillas del Orinoco por Joseph Yturriaga, comandante de la Comisión de Límites de 1750. No obstante el aumento de precisión y los detalles con que Alcedo presenta la investigación asociada al Orinoco, la narración termina por ubicar el nacimiento del Orinoco en una imaginaria Sierra Nevada situada en la Provincia de Guayana, al norte del Parima; ello, según señala, apoyándose en los resultados de la Expedición de Límites y en los informantes Caribes. Se mantenía entonces una idea sobre la existencia de una cadena montañosa continua que iría de los Andes a la Cayena y que motivaría más tarde un debate entre Buache y Humboldt (Debarbieux, 2008). El lugar de las fuentes seguía (aunque esta vez sí orientada a la Guayana) mostrando las dificultades de su real emplazamiento y gobernando el régimen de la imaginación geográfica proyectada a las terra incognitae y la construcción de un territorio posible, pues se pensaba que más allá, en sus confines, se escondían grandes riquezas.

En una visión de conjunto, la imaginación geográfica del Orinoco y de la Guayana estuvo gobernada por discursos y representaciones cuyas narrativas y cartografías enlazaban dos formas de conocer: de un lado, el mapa; del otro, el diccionario geográfico y las historias naturales y corográficas. Estos textos creaban la ilusión de conocer y con ello la de territorializar mediante la proyección de poblamientos que impulsasen el progreso de estos territorios de conformidad con la imagen emergente del mundo. 


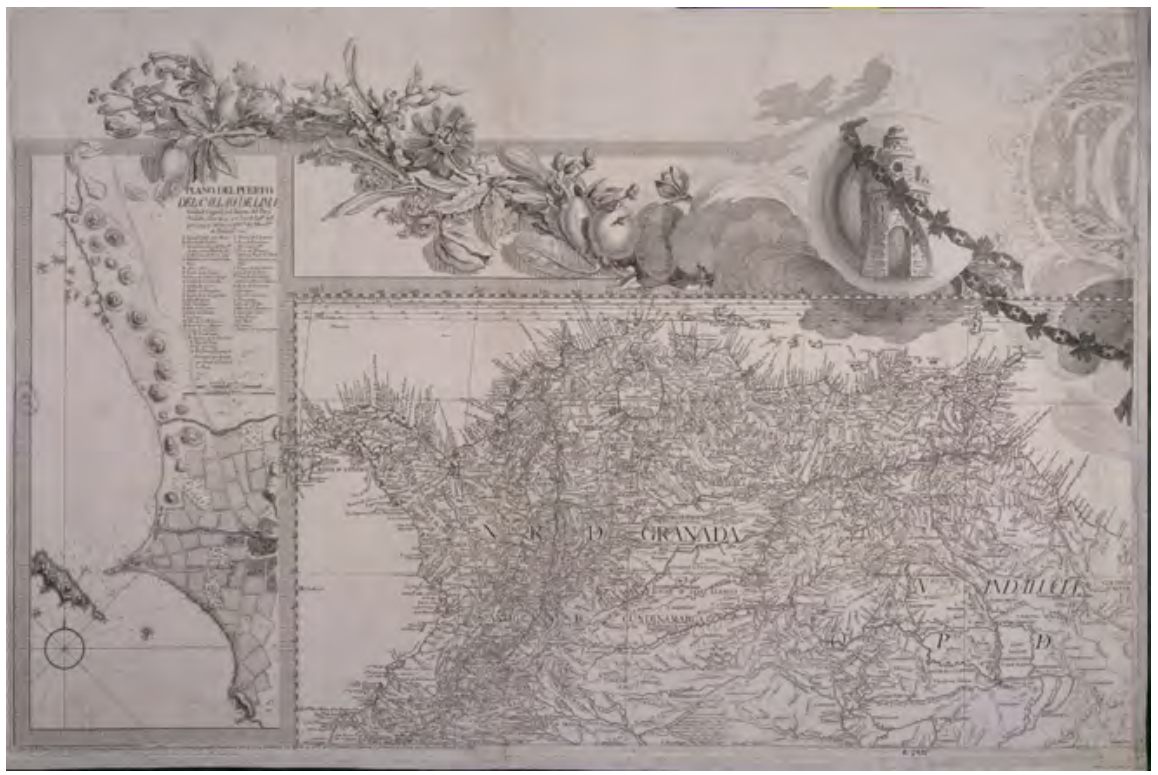

\section{FIGURA 2.}

Mapa geográfico de América

Meridional (grabado $\mathrm{n}^{\circ} 1$ ),

de Juan de la Cruz, Cano y

Olmedilla (1775).

Fuente: Fondos de la Biblioteca

Nacional de España.

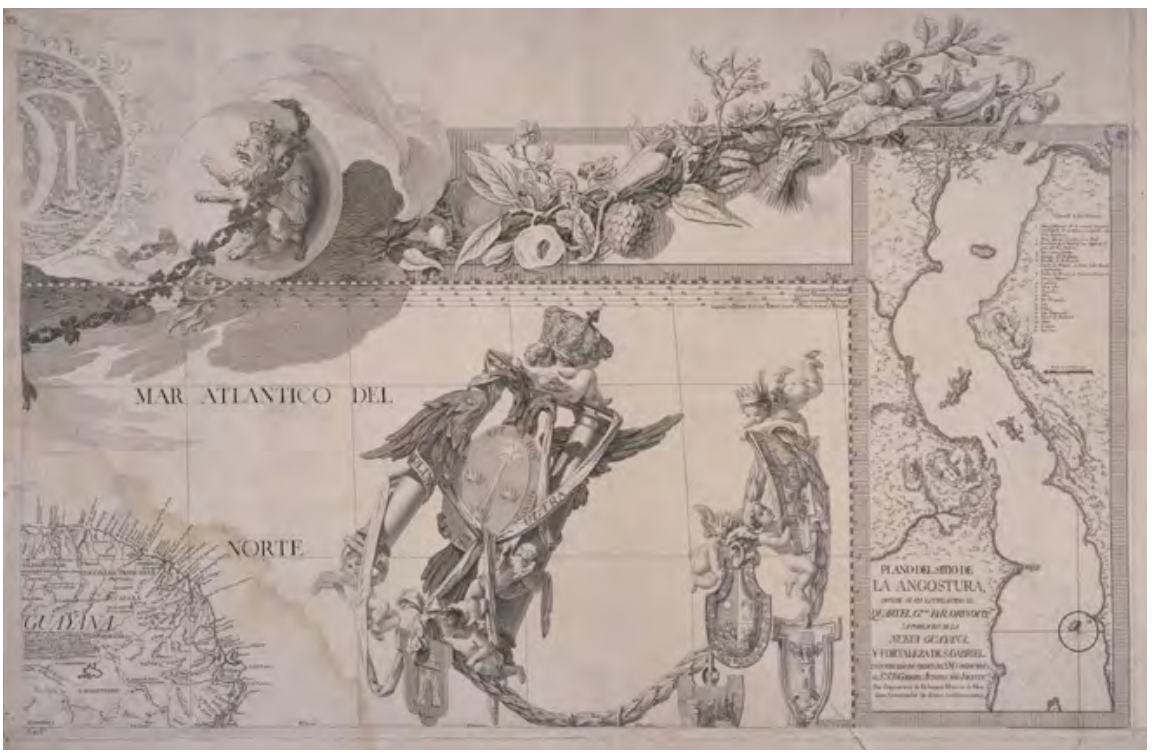

FIGURA 3.

Mapa geográfico de América

Meridional (Grabado n 2

con el "Plano del sitio de

la Angostura donde se ha establecido el Quartel Gral. De R. Orinoco, la población de la Nueva Guayana y Fortaleza de S. Gabriel, construida de orden de S.M. y dedicada al S. Sr. D. Gabriel Antonio Nro. Infante, por disposición de D. Juan Moreno de Mendoza, Governador de dichos establecimientos"), de Juan de la Cruz, Cano y Olmedilla. (1775).

Fuente: Fondos de la Biblioteca Nacional de España.

Este trabajo de los observadores del Orinoco y la Guayana construyó un texto espacial que autorizó performances localizadas (Livingstone, 2002), bases de una experiencia cognitiva que enlazaba episteme y praxis, pero también una erótica espacial que creaba ilusiones en las fronteras. En tal contexto, los mapas podían mentir, como señala Monmonier (1996), podían imaginar cosas que no estaban, como lagos míticos y cadenas montañosas continuas, dibujar limes no conocidos y una geografía humana ambivalente, pero no es menos cierto que esos dibujos de las terrae incongnitae 
abrieron nuevos derroteros para el nuevo régimen de exploraciones y viajes al interior del continente, que ocuparían la imaginación geográfica del siglo por venir y se convertirían en correlatos de atracción para el trabajo de territorialización en el binomio de poblamiento y progreso, según los marcos de la geografía política emergente del diseño europeo y la experiencia del espacio y los lugares de América.

Hacia fines del siglo XVIII algunos poblamientos en la Guayana, producto de la acción de misioneros y de funcionarios del reformismo español, no estaban consolidados, salvo en algunos emplazamientos como Angostura, Cabruta y Caicara. A lo largo de las riberas del alto Orinoco y a partir del fortín de Marimarota hasta la red de fortines en el área de convergencia hidrográfica del Orinoco, Atabapo, Guainía-Río Negro subsistían formas de poblamiento incipientes que resistían las duras condiciones de la frontera imperial.

Desde este punto de vista, la implantación del modelo colonial, si bien creó ilusiones de poblamiento, no pudo vencer los obstáculos de clima, técnica y oposición étnica, lo que marcó la historia republicana durante el siglo XIX y hasta mediados del siglo XX. Estos imaginarios geográficos de la frontera nos hablan hoy día de una región en donde, a pesar de las riquezas, los procesos de territorialización aún no se han consolidado. Aquí el espacio, como dice Claval (2013), tiene una historia móvil, una historia de flujos y discontinuidades y también de permanencias, una cuestión observable en los espacios periféricos vinculados al río Orinoco y la Guayana, cuyas densidades demográficas son muy bajas y solo están concentradas en Ciudad Bolívar y Ciudad Guayana.

Sin embargo, en los momentos actuales, sufren el expolio minero y de otros recursos naturales, acelerados a partir de 2016 por el Decreto 2248 de creación de la Zona de Desarrollo Estratégico Nacional "Arco Minero del Orinoco" (Presidencia de la República Bolivariana de Venezuela, 2016). Este decreto ha generado, como se sabe, protestas de las etnias que habitan esos territorios y entornos naturales y de los movimientos ciudadanos preocupados por el impacto ambiental, ya que su expresión visible es la de un horizonte de destrucción de los ecosistemas y de los medios de vida de muchas de las etnias originarias de esa región.

\section{Agradecimiento}

El desarrollo de esta investigación y su resultado se hizo dentro del proyecto Conacyt-México: Territorios y Altergeografías. Tercer espacio, poder y percepciones de la vida en común. Universidad Autónoma Metropolitana-Xochimilco, Doctorado en Ciencias Sociales, área Sociedad y Territorio. 


\section{Referencias}

Alcedo, A. (1788). Diccionario geográfico-histórico de las Indias Occidentales o América: es a saber: de los Reynos del Perú, Nueva España, Tierra Firme, Chile y Nuevo Reyno de Granada. Con la descripción de sus Provincias, Naciones, Ciudades, Villas, Pueblos, Ríos, Montes, Costas, Puertos, Islas, Arzobispados, Obispados, Audiencias, Virreynatos, Gobiernos, Corregimientos, y Fortalezas, frutos y producciones; con expresión de sus Descubrimientos, Conquistadores y Fundadores: Conventos y Religiones; erección de sus Catedrales y Obispos que ha habido en ellas: Y noticia de los sucesos más notables de varios lugares: incendios, terremotos, sitios, é invasiones que han experimentado: $y$ hombres ilustres que han producido. (Vol. 3). Madrid, España: Imprenta de Blas Román. Recuperado de https://archive.org/search. php?query $=$ creator $\% 3 \mathrm{~A} \% 22 \mathrm{Alcedo} \% 2 \mathrm{C}+$ Antonio + de $\% 2 \mathrm{C}+1735-1812 \% 22$

Alvarado, E. (1999). Apuntes para una descripción geográfica de lo que debe ser la Provincia de Guyana en toda su extensión y sus límites, conforme a la línea divisoria que debió tirarse entre los dominios de España y Portugal, por E. Alvarado. En M. Lucena G., Viajes a la Guayana ilustrada: el hombre y el territorio (Estudio introductorio, selección y notas de M. Lucena Giraldo), (pp. 149-154). Caracas, Venezuela: Banco Provincial.

Amodio, E. (1998). El paraíso vegetal. Las fronteras étnicas de un botánico ilustrado. En M. San Pío Aladren (coord.), La Comisión naturalista de Löfling en la Expedición de Límites al Orinoco (pp. 61-84). Madrid, España: Real Jardín Botánico, Consejo Superior de Investigaciones Científicas, Lunwerg Editores.

Bellin, J. N. (1986). Descripción geográfica de la Guayana. Contiene las posesiones y establecimiento de los franceses, de los españoles y de los holandeses en estas vastas comarcas. [Con mapas desplegables]. Caracas, Venezuela: Ediciones de la Presidencia de la República.

Brendeke, A. (2016). Imperio e información. Funciones del saber en el dominio colonial español. (2a ed. ampliada). Madrid, España; Fráncfort, Alemania: Iberoamericana, Vervuert Verlag.

Bueno, B. P. S. (2011). Desenho e designio, o Brasil dos engenheiros militares (15001882). São Paulo, Brasil: Editora da Universidade de São Paulo.

Capel, H. (2003). Los diccionarios geográficos de la ilustración española. En J. Moncada (coord.), La geografía de la llustración (pp. 41-156). México: Instituto de Geografía, Universidad Nacional Autónoma de México.

Capel, H., Moncada, J., García, L., Olivé, F. Quesada, S., Rodríguez, A. ...Tello, R. (1983). Los Ingenieros militares en España, siglo XVIII: repertorio biográfico e inventario de su labor científica y espacial. Barcelona, España: Universitat de Barcelona, Edicions i Publicacions, Geo-crítica.

Carrocera, B. (1979). Misión de los capuchinos en Guayana. (3 vols.). Caracas, Venezuela: Academia Nacional de la Historia. 
Claval, P. (2013). ¿Como construir a história da geografia? Terra Brasilis (Nova Série), 2. 1-23. Recuperado de https://journals.openedition.org/terrabrasilis/637.

Cuevas Quintero, L. M. (2012). Como el río que fluye: los jesuitas en el Orinoco, producciones de espacialidad y experiencia de lugares en el siglo XVIII. (Tesis de maestría). Universidad Iberoamericana, México D. F., México.

Cuevas Quintero, L. M. (2017a). Navegando en el mar de los gentiles. Metáforas del espacio sagrado en el discurso jesuita del Orinoco (siglo XVIII). Revista Geográfica Venezolana, 58(1), 222-239.

Cuevas Quintero, L. M. (2006). Percepción y discurso geográfico sobre la Orinoquia: la invención del espacio en Joseph Gumilla. (Trabajo de Ascenso). Universidad de Los Andes, Mérida, Venezuela.

Cuevas Quintero, L. M. (2017b). El soberbio Orinoco, Viajes, Ciencia e imaginación geográfica, 1799-1951. (Tesis doctoral). México: Universidad Nacional Autónoma de México, México D. F., México.

Dardel, E. (1952). L’homme et la terre: nature de la realité geographique. París, Francia: Presses Universitaires de France, Nouvelle encyclopédie philosophique.

De Barcelona, Fray C. (1779). Sketch Map of the Missions of the Catalonia Capuchins in the Spanish Province of Guayana. David Rumsey Historical Map Collection. Recuperado de https://www.davidrumsey.com/luna/servlet/detail/RUMSEY 8 1 204086 30 01793:Capuchin-Missions-of-Guayana-by-Car?

De la Cruz, Cano y Olmedilla, J. (1775). Mapa geográfico de América Meridional (Grabado n. 1). Fondos de la Biblioteca Nacional de España.Recuperado de http:// bdh-rd.bne.es/viewer.vm?id=0000051497.

De la Cruz, Cano y Olmedilla, J. (1775). Mapa geográfico de América Meridional. (Grabado n. 2 con el "Plano del sitio de la Angostura donde se ha establecido el Quartel Gral. De R. Orinoco, la población de la Nueva Guayana y Fortaleza de S. Gabriel, construida de orden de S.M. y dedicada al S. Sr. D. Gabriel Antonio Nro. Infante, por disposición de D. Juan Moreno de Mendoza, Governador de dichos. Establecimientos"). Fondos de la Biblioteca Nacional de España. Recuperado de http://bdh-rd.bne.es/ viewer.vm?id.

Debarbieux, B. (2008), Mountains between corporal experience and pure rationality: Buache and Von Humboldt's contradictory theories. En D. Cosgrove \& V. Della Dora (edits.), High Places. Cultural Geographies of Mountains, Ice and Science (pp. 87-104). London, United Kingdom: I. B. Tauris.

Del Rey Fajardo, J. (1975). Apuntes para una historia de la cartografía jesuítica en Venezuela. Boletín Histórico, Fundación John Boulton, 38, 152-170.

Del Rey Fajardo, J. (2011). Los hombres de los ríos. Suplemento Encuentro de Provincia, 7, 2-23.

Del Rey Fajardo, J. (1998). Una utopía sofocada: reducciones jesuíticas en la Orinoquia. Caracas, Venezuela: Universidad Católica Andrés Bello.

Denevan, W. (1992). The Pristine Myth: The Landscape of the Americas in 1492. Annals of the Association of American Geographers, 82(3), 369-385. 
Donís Ríos, M. (1997). Guayana: historia de su territorialidad. Caracas, Venezuela: Universidad Católica Andrés Bello, Instituto de Investigaciones Históricas, Ferrominera del Orinoco.

Gumilla, J. (1983). El Orinoco ilustrado y defendido. Caracas, Venezuela: Biblioteca de la Academia Nacional de la Historia.

Hartog, F. (2007). Regímenes de historicidad. México: Universidad Iberoamericana.

Inciarte, J. F. (1968). Informe de 5 de diciembre de 1783 del Oficial José Felipe Inciarte sobre la parte Oriental del Bajo Orinoco que tenía a su cuidado. En J. de Armas Chitty, Guayana, su tierra y su historia (t. II, pp. 330-335). Caracas, Venezuela: Dirección de Cartografía Nacional del Ministerio de Obras Públicas.

Lacotoure, J. (1993). Jesuitas: los conquistadores. (T. I). Barcelona, España: Paidós.

Livingstone, D. N. (2004). Cultures of science. En J. Duncan, N. Johnson \& R. Schein (eds.), A Companion to Cultural Geography (pp. 139-150). London, United Kingdom: Blackwell Publishing.

Livingstone, D. N. (2002). Science, Space and Hermeneutics, The Hettner-Lectures 2001. Heildelberg, Alemania: Department of Geography, University of Heidelberg.

Lucena Giraldo, M. (1998). El Dorado geométrico. La Expedición de Límites al Orinoco 1754-1761. En M. San Pío Aladren (coord.), La Comisión naturalista de Löfling en la Expedición de Límites al Orinoco (pp. 23-40). Madrid, España: Real Jardín Botánico, Consejo Superior de Investigaciones Científicas, Lunwerg editores.

Lucena Giraldo, M. (1993). Laboratorio tropical. La expedición de límites al Orinoco 1750-1767. Caracas, Venezuela: Monte Ávila Editores Latinoamericana, Consejo Superior de Investigaciones Científicas.

Lucena Giraldo, M. (1992). La última búsqueda de El Dorado: las expediciones al Parime (1770-1776). Iberoamericana Pragensia, 21, 67-86.

Lucena Giraldo, M. (1999). Viajes a la Guayana ilustrada: el hombre y el territorio. (Estudio introductorio, selección y notas de M. Lucena Giraldo). Caracas, Venezuela: Banco Provincial.

Lucena Giraldo, M. \& De Pedro, A. (1992). La frontera caríbica: expedición de límites al Orinoco, 1754/1761. Caracas, Venezuela: Lagoven.

Ludlow, L. \& Martínez de López Ocón, M. (Coords.) (2007). Historia del liberalismo económico: del mercantilismo al liberalismo. México: Instituto de Investigaciones Históricas, Universidad Autónoma de México, Instituto Mora.

Lynch, J. (1987). Hispanoamérica, 1750-1850: ensayos sobre la sociedad y el Estado. Bogotá, Colombia: Universidad Nacional de Colombia.

Mandrini, R. (1992). Indios y fronteras en el área pampeana (siglos XVI-XIX): balance y perspectivas. Anuario del IEHS, 7, 59-73.

Marmión, M. (1999). Descripción corográfica-mixta de la provincia de Guayana en que se da razón de los ríos que la bañan y facilitan sus comunicaciones: de su 
población, tierras de labor útiles, de sus frondosos montes, frutos y comercio, y se proponen algunos medios los más asequibles y conducentes a su verificación y aumento, 1788. En M. Lucena, Viajes a la Guayana ilustrada. El hombre y su territorio (pp. 245-264). Caracas, Venezuela: Banco Provincial.

Merino, O. \& Newson, L. (1995). Jesuit Missions in Spanish America: The Aftermath of The Expulsion. En Yearbook (Conference of Latin Americanist Geographers) (vol. 21, pp. 133-148). Austin, EE. UU.: University of Texas Press.

Monmonier, M. (1996). How to Lie with Maps. Chicago, EE. UU., London, United Kingdom: The University of Chicago Press.

Perera, M. (2006). El Orinoco domeñado. Frontera y límite. Guayana siglo XVIII, ecología cultural y antropología histórica de una colonización breve e inconclusa. Caracas, Venezuela: Universidad Central de Venezuela, Consejo de Desarrollo Científico y Humanístico.

Presidencia de la República Bolivariana de Venezuela. (2016). Decreto 2248, mediante el cual se crea la Zona de Desarrollo Estratégico Nacional "Arco Minero del Orinoco". Gaceta Oficial de la República Bolivariana de Venezuela n 40855. Recuperado de http://historico.tsj.gob.ve/gaceta/febrero/2422016/2422016-4514. pdf

Ramos Pérez, D. (1946). Tratado de Límites de 1750 y la Expedición de Iturriaga al Orinoco. Madrid, España: Consejo Superior de Investigaciones Científicas.

Ryden, S. (1957). Pedro Loefling en Venezuela (1754-1756). Madrid, España: Ínsula.

Weber, D. (2007). Bárbaros: los españoles y sus salvajes en la era de la Ilustración. Barcelona, España: Crítica. 